\title{
ThalPred: a web-based prediction tool for discriminating thalassemia trait and iron deficiency anemia
}

\author{
V. Laengsri ${ }^{1,2}$, W. Shoombuatong ${ }^{3}$, W. Adirojananon ${ }^{2}$, C. Nantasenamat ${ }^{3}$, V. Prachayasittikul ${ }^{4}$ and P. Nuchnoi ${ }^{1,2^{*}}$ (D)
}

\begin{abstract}
Background: The hypochromic microcytic anemia (HMA) commonly found in Thailand are iron deficiency anemia (IDA) and thalassemia trait (TT). Accurate discrimination between IDA and $T$ is an important issue and better methods are urgently needed. Although considerable RBC formulas and indices with various optimal cut-off values have been developed, distinguishing between IDA and TT is still a challenging problem due to the diversity of various anemic populations. To address this problem, it is desirable to develop an improved and automated prediction model for discriminating IDA from $\Pi$.

Methods: We retrospectively collected laboratory data of HMA found in Thai adults. Five machine learnings, including $k$-nearest neighbor ( $k-N N)$, decision tree, random forest (RF), artificial neural network (ANN) and support vector machine (SVM), were applied to construct a discriminant model. Performance was assessed and compared with thirteen existing discriminant formulas and indices.

Results: The data of 186 patients (146 patients with $\Pi$ and 40 with IDA) were enrolled. The interpretable rules derived from the RF model were proposed to demonstrate the combination of RBC indices for discriminating IDA from $\Pi$. A web-based tool 'ThalPred' was implemented using an SVM model based on seven RBC parameters. ThalPred achieved prediction results with an external accuracy, MCC and AUC of 95.59, 0.87 and 0.98, respectively.

Conclusion: ThalPred and an interpretable rule were provided for distinguishing IDA from $\Pi$. For the convenience of health care team experimental scientists, a web-based tool has been established at http://codes.bio/thalpred/ by which users can easily get their desired screening test result without the need to go through the underlying mathematical and computational details.
\end{abstract}

Keywords: Thalassemia trait, Iron deficiency anemia, Machine learning, Support vector machine, Random forest, Discrimination, Decision making

\section{Background}

Anemia is the condition of decreased number of red blood cells (RBCs) or of the concentration of hemoglobin $(\mathrm{Hb})$. Anemia is a health problem affecting both developing and developed countries. The global prevalence of anemia in 2010 was $32.9 \%$ and it was especially common in Central Africa, the Middle East, the Mediterranean and Southeast

\footnotetext{
* Correspondence: pornlada.nuc@mahidol.ac.th

${ }^{1}$ Center for Research and Innovation, Faculty of Medical Technology, Mahidol University, Bangkok, Thailand

${ }^{2}$ Department of Clinical Microscopy, Faculty of Medical Technology, Mahidol

University, Bangkok, Thailand

Full list of author information is available at the end of the article
}

Asia [1]. It can occur from one of three causes, acute blood loss, increased hemolysis or ineffective hematopoiesis.

In Thailand, both iron deficiency anemia (IDA) and thalassemia trait (TT) are highly prevalent. Iron is an important element in our body, being a component of many enzymes and playing a role in hemoglobin synthesis. Therefore, a lack of iron can lead to IDA. The prevalence of IDA among Thai people is estimated to be $1.5-8 \%$ [2]. Thalassemia is an inherited hematological disorder that is caused by abnormal production of alpha $(\alpha)$ - or beta $(\beta)$ globin chains. The prevalence of TT is approximately 20 $25 \%$ in the Thai population [3]. The levels of serum ferritin, serum iron, total iron binding capacity and 
percentage of transferrin saturation are the most commonly used assays to confirm IDA [4]. Meanwhile, Hb testing via high performance liquid chromatography or capillary electrophoresis to detect abnormal $\mathrm{Hb}$ levels or DNA analysis are the assays commonly used for TT diagnosis [5-7]. In practice, these latter techniques are not available in routine laboratories as they require special machines, and are time- and cost-consuming. Basically, when patients were assumed to be TT or IDA, clinician often prescribed the cassette of hematological tests covering both TT and IDA diagnosis. These laboratory workups consume personnel work load and governmental budget. This leads to financial crisis for national health care of low-middle income countries. In order to guide clinician for rational lab use, we therefore developed webbased tool for assisting clinician to prescribe rational and cost-effective laboratory testing for TT and IDA diagnosis. Multiple formulas and indices have been proposed for such discrimination including the Bessman index (BI) [8], Ehsani formula (EF) [9], England \& Fraser index (E\&F) [10], Green \& King index (G\&K) [11], Mentzler index (MI) [12], Red Cell Distribution Width index (RDWI) [13], Ricerca index (RI) [14], Shine and Lal index (S\&L) [15], Siridah index (SI) [16], Srivastava formula (SF) [17], Sirachainan formula (SiF) [18], Kandhro 1 formula (KF1) [19] and Kandhro 2 formula (KF2) [19].

The aforementioned discriminant indices and formulas yielded quite encouraging prediction results. However, the prediction results across different populations, especially in sensitivity and specificity, are still unsatisfactory. Some of studies are contentious due to difference of gender, age or ethnicity [20-22]. In order to improve prediction results, the computation of optimal cut-off values for specific populations in different countries is needed [23, 24]. The potential of machine learning techniques has been demonstrated for near-term translational impact. For instance, in the case of the biomedicine, major applications of machine learning are medical/radiological diagnosis and drug discovery. Thus, it may be possible to develop discriminant models based on machine learning techniques for providing effective large-scale analyses of laboratory data. To the best of our knowledge, only one discriminant model has been proposed to differentiate IDA and TT in the Thai population [18]. In this study, we exerted an effort to develop a powerful model to discriminate IDA from TT using a support vector machine (SVM) which we named ThalPred.

The previous works had demonstrated the meaningful of data mining and the increasing of computational power in various aspect of biomedical application [25-28]. The present study aims to establish a reliable and interpretable computational model. Therefore, the important procedures as the following are considered: (i) collect clear and reliable laboratory dataset for training and validating; (ii) demonstrate characteristic or descriptor of dataset for intrinsic properties prediction; (iii) identify feature of importance for improving interpretability; (iv) develop a simple and interpretable model; (v) perform rigorous cross validation for analyzing internal and external predictive power; (vi) develop affordable and user friendly based web tool for implementation in the healthcare community.

\section{Material and methods \\ Data collection}

This was a retrospective study of encoded and unlinked clinical laboratory data obtained from the Center of Medical Laboratory Services, the Faculty of Medical Technology, Mahidol University from the period July 2014 to September 2016. The abbreviations of hematological laboratory testing were listed in Table 1 . We collected 237 sets of hematological data from Thai subjects, age 18 to 60 years, that showed hypochromic microcytic anemia (HMA) and were diagnosed as reflecting IDA or TT by two independent medical technologists. Other causes of HMA, such as hypothyroidism, anemia of chronic disorders, hepatitis B / C / or D infection, or Helicobacter pylori infection, were excluded from the study in order to specify IDA and TT only. Hemoglobin electrophoresis and serum ferritin results were analyzed to discriminate TT and IDA, respectively. The level of ferritin was determined by elec-

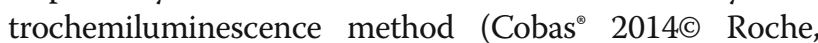
Switzerland). Variant hemoglobin testing was performed using low pressure liquid chromatography (Bio-Rad Laboratories, USA). The final dataset consisted of 186 subjects (146 TT and 40 IDA cases) which were used for internal and external analyses. The study was conducted under the approval of the Mahidol University Central Institutional Review Board (MU-CIRB; CODE No. MUCIRB 2016/084.0311). We received a participant consent waiver from MU-CIRB. All information of subjects was de-identified prior data analysis.

Table 1 List of laboratory testing abbreviation used in this study

\begin{tabular}{ll}
\hline Full Name & Abbreviation \\
\hline Hemoglobin & $\mathrm{Hb}$ \\
Hematocrit & $\mathrm{Hct}$ \\
Hypochromic microcytic anemia & $\mathrm{HMA}$ \\
Iron deficiency anemia & $\mathrm{IDA}$ \\
Mean corpuscular volume & $\mathrm{MCV}$ \\
Mean corpuscular hemoglobin & $\mathrm{MCH}$ \\
Mean corpuscular hemoglobin concentration & $\mathrm{MCHC}$ \\
Red blood cell & $\mathrm{RBC}$ \\
Red blood cell distribution width & $\mathrm{RDW}$ \\
Thalassemia trait & $\mathrm{TT}$ \\
\hline
\end{tabular}




\section{Statistical analyses}

The statistical analysis of this study was computed using SPSS software, version 20 (SPSS Inc., New York, Armonk, USA). As an exploratory statistical analysis, univariate statistical analysis using mean and standard deviation (SD) was performed to investigate the different patterns and trends of individual hematological parameters between the data of groups TT and IDA. The KolmogorovSmirnov test was used for normality testing. Since the data of this study was not normally distributed. The comparison between groups TT and IDA was performed by using the Mann-Whitney $\mathrm{U}$ test. A $p$-value $<0.05$ was considered significant. Furthermore, in order to perform multivariate statistical analyses amongst $\mathrm{RBC}$ indices, principal component analysis (PCA) was performed. PCA has probably been the most popular technique to perform multivariate statistical analysis for the tasks of data exploration and pattern recognition. The advantages of PCA are to: (i) extract the most important information and represent it with only a few dimensions, called principal components (PCs); (ii) compress the dimensions of the dataset by keeping only the important information; and (iii) analyze the characteristics and structure of the data set. In this study, PCA was performed by using the FactoMineR package [29] in R program to represent the distributions of IDA and TT cases, and identify the RBC indices important for distinguishing IDA and TT cases.

\section{Models construction}

The following five popular computational models, namely $k$-nearest neighbor $(k-\mathrm{NN})$, decision tree (DT), random forest (RF), artificial neural network (ANN) and support vector machine learning (SVM), were applied to develop discriminant models for effectively distinguishing TT from IDA. The fundamental and associated parameter optimization for the five classifiers are briefly described (Fig. 1).

$k$-NN is one of the most popular and lazy machine learning algorithms for a variety of problem domains $[25,30,31]$. This algorithm is conceptually based on a distance function, such as the Euclidean distance, to measure the similarity between a pair of training and unknown datasets. For obtaining the best $k$-NN model, the suitable number of neighbors $(k \in\{5,7,9, \ldots, 43\}$ was determined by using the caret package $[28,32]$ over 5 -fold cross-validation (5-fold CV) scheme.

DT model can be used in the classification and regression tasks by constructing a model in the form of a tree structure. Herein, we constructed the DT model by implementing the J48 algorithm from the RWeka package in R program (version 3.3.2) [33] using default parameters. Briefly, the J48 algorithm is a re-implementation of the C4.5 algorithm [34] based on Javascript. The feature with the highest information gain is select to build a model. Finally, because of its built-in feature selector, the DT

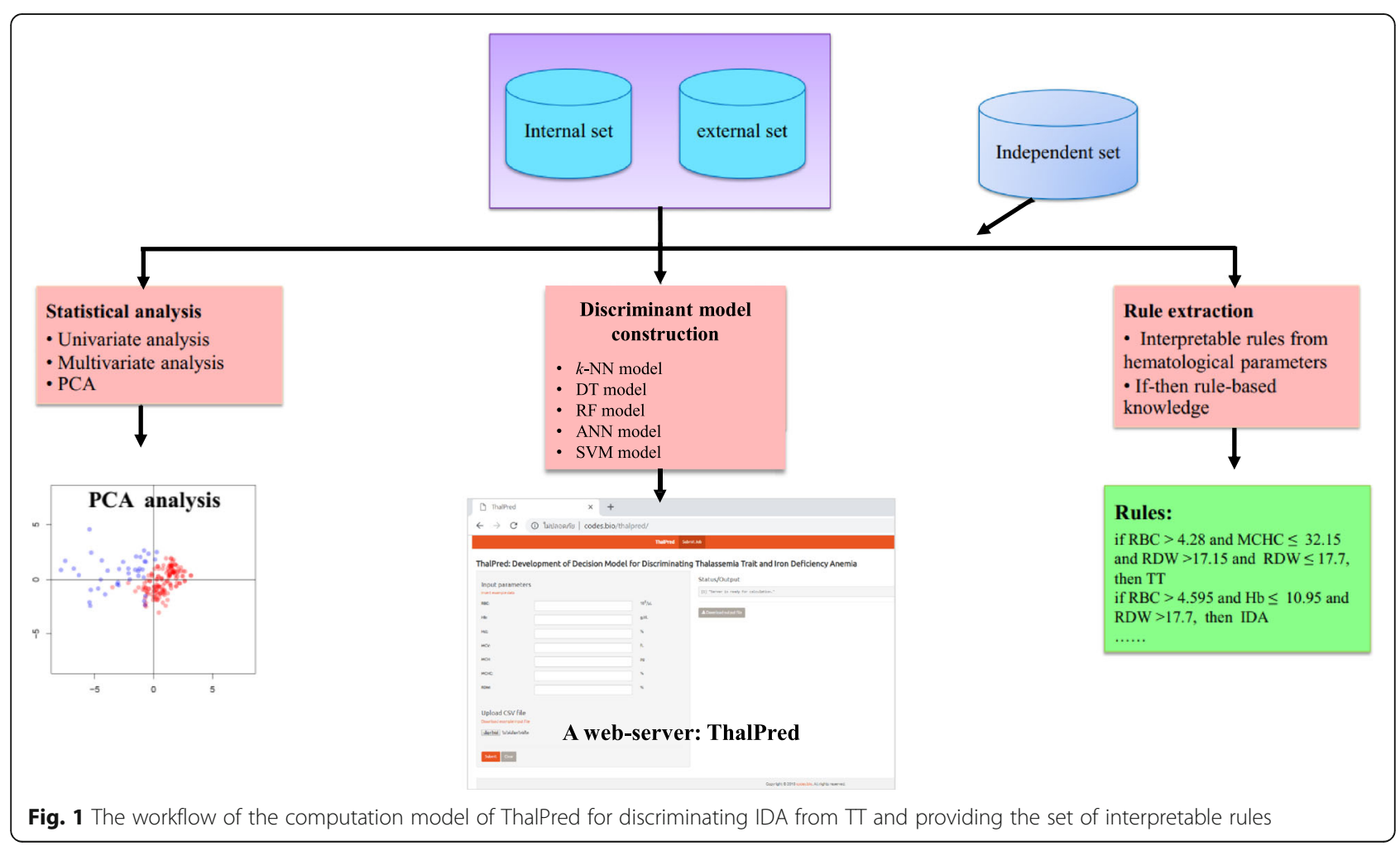


model will provide the feature usage score for ranking the feature importance.

$\mathrm{RF}$ is an ensemble classification and regression tree (CART) classifier [34, 35]. The RF model is a classifier derived from gathering many weak CART trees for improving the prediction performance. To construct the model, RF takes advantage of two well-known machine learning algorithms, i.e. bagging and random feature selection. To estimate informative features, RF model utilizes two measures, i.e. mean decrease in accuracy and Gini index [35]. In this study, the RF classifier was implemented using the randomForest $R$ package [34]. To obtain an optimal RF model, two parameters, namely, ntree (i.e., the number of trees used for constructing the RF classifier) and mtry (i.e., the number of random candidate features), were tuned by using a grid search procedure based on 5-fold cross-validation (5-fold $C V)$, where ntree $\in\{100,200,300,400,500\}$ was determined, while mtry was estimated using the tuneRF function in the randomForest $\mathrm{R}$ package [34].

ANN is computing systems originally inspired by the way biological nervous systems process information [36]. Previously, many researchers reported that ANN accomplished well in many domains, such as protein sequence analysis, image recognition, speech recognition, and natural language processing $[27,37,38]$. In practice, there are two important types of ANN, i.e. the perceptron and the sigmoid neuron, while stochastic gradient descent is known as the standard estimating parameter algorithm for ANN. For achieving the best ANN model, two parameters, namely, size (i.e., the number of hidden nodes) and decay (i.e., the number of weight decay), were subjected to optimization. Particularly, size $\in\{1,2,3, \ldots, 10\}$ and decay $\in\{0.1,0.2,0.3,0.4,0.5\}$ were determined by using the caret package $[28,32]$ over a 5 -fold CV scheme.

SVM is a statistical learning approach based on the principle of structure-risk minimization and a kernel method (as proposed by Vapnik [39]) which are used to construct a maximum-margin-separating hyperplane for distinguishing the two classes of interest. The radial basis function kernel was used to transform the original feature space into a higher dimensional space in which the SVM classifier can linearly separate the inherent classes of the dependent variable via a maximum separating hyperplane [40]. Optimization of the SVM parameters consisting of the cost $\in\left\{2^{8}, 2^{7}, 2^{6}, \ldots, 2^{7}, 2^{8}\right\}$ and $\gamma$ $\in\left\{2^{8}, 2^{7}, 2^{6}, \ldots, 2^{7}, 2^{8}\right\}$ were determined via a grid search spanning the search space evaluated by 5 -fold $\mathrm{CV}$ scheme using the e1071R package [41].

\section{Cross-validation for identification of discriminant capability}

The validation of an empirical predictive model is essential. In order to train and evaluate the discriminant models, the data set of this study was randomly partitioned into internal and external sets with 80 and $20 \%$, respectively, of the data set from both TT and IDA. The internal set was evaluated using the 5-fold CV scheme [42] to confirm the reliability and robustness of the proposed discriminative model. The external set was used to assess the generalizability of the model when extrapolating to unknown samples. To avoid the possibility of bias arising from a single data split upon model training, data splitting was performed for 100 independent iterations. The final prediction performances of the 5 -fold $\mathrm{CV}$ and external validation tests of the proposed discriminative model were reported by using the mean and standard deviation values of analyzed parameters (Fig. 1).

\section{Rule extraction}

In addition to model accuracy and ability to discriminate IDA from TT, there is a possibility that a simple and meaningful rule may be extracted from an RF model. From the nroot to a leaf node is a rule for a tree. The main purpose of extraction rules is to ease utilization and make the model more interpretable compared to black-box approaches [25] such as SVM. In this study, the extraction rules were obtained by using the $\mathrm{R}$ package inTrees (interpretable trees) [34] and only 100 decision trees were established for each encoding to train the RF model. InTrees is a powerful package for extracting, measuring, pruning, selecting and summarizing from an RF model (Fig. 1).

\section{Performance assessment}

Performance assessment is important step for developing reliable and useful predictor. In order to discriminate TT from IDA, pattern recognition is applied for classification concept. Five standard statistical parameters, namely, accuracy (Ac), sensitivity (Sn), specificity (Sp), Matthew's correlation coefficient (MCC), and Youden's index (YI), were addressed to evaluate the predictive performance of the proposed methods. These five parameters were computed as follows [43-46]:

$$
\begin{aligned}
& \mathbf{A c}=\frac{T P+T N}{(T P+T N+F P+F N)} \times 100 \\
& \mathbf{S n}=\frac{T P}{(T P+F N)} \times 100 \\
& \mathbf{S} \mathbf{p}=\frac{T N}{(T N+F P)} \times 100 \\
& \mathbf{M C C}=\frac{(T P)(T N)-(F P)(F n)}{\sqrt{(T P+F P)(T P+F N)(T N+F P)(T N+F N)}} \\
& \mathbf{Y I}=[(\mathrm{Sn}+\mathrm{Sp})-100] / 100
\end{aligned}
$$

where TP, FP, TN and FN are true positive, false positive, true negative and false negative, respectively. Furthermore, 
the receiver operating characteristic (ROC) curve, which plots the true positive rate (Sn) against the false positive rate (1-Sp) at different thresholds, was also used for model evaluation. A predictor with perfect classification has a ROC curve passing through the top-left corner, i.e. $\mathrm{Sn}=$ $100 \%$ and $\mathrm{Sp}=100 \%$. To plot the ROC curve and calculate the value of area under the curve (AUC), the ROCR package [47] was used.

\section{Results}

Differences in hematological data of TT and IDA using univariate and multivariate analyses

HMA data was classified into two groups: 146 TT cases and 40 IDA cases. The characteristics and hematological data of both groups of IDA and TT are summarized in Table 2. Age of two groups was not significantly different ( $p$-value 0.435$)$. All RBC parameters were significantly different between the two groups $(p<0.05)$. All averages of parameters in TT group were higher than IDA group except RDW. Furthermore, PCA was applied as multivariate analysis was presented as scores (Fig. 2a) and loadings plots (Fig. 2b). The percentage of variance explained by the first two PCs (83.33\%) were considered sufficient in describing the behavior of the data. The graphs of Fig. 2a and Fig. 2b also revealed that RDW contributes greatly to IDA, while the remaining $\mathrm{RBC}$ parameters contribute to TT. These results were well reflected by the mean values of IDA $(15.88 \pm 1.13)$ and TT (20. $48 \pm 3.23)$. Interestingly, the outputs of two analyses were consistent. Therefore, these suggested that all RBC indices may be important to discriminate between patients with IDA and those with TT.

\section{Evaluation of existing discriminant formulas and indices}

To objectively evaluate the performance of our proposed discriminant model and fairly compare it with existing discriminant formulas and indices including BI [8], EF [9], E\&F [10], G\&K [11], MI [12], RDWI [13], RI [14],
S\&L [15], SI [16], SF [17], SiF [18], KF1 [19] and KF2 [19], the same dataset was used for evaluation of each. Their cut-off, Ac, Sn, Sp, MCC, YI, and AUC were shown in Table 3.

As noticed in Fig. 3, most existing formulas and indices achieved an AUC value that was greater than 0.5 [except for S\&L (0. 31) and SiF (0.02)]. The highest MCC and AUC of 0.74 and 0.98 , respectively, were achieved by the KF2 formula. Meanwhile, RI and G\&K formulas performed well with the second and third highest MCC/ AUC of $0.70 / 0.98$ and $0.66 / 0.93$, respectively. Meanwhile, the formulas and indices with the lowest values of MCC/AUC were SiF, S\&L and EF (-05.6/0.02, 0.01/0. 31 and $0.17 / 0.70$, respectively). These prediction performances indicated that only a few formulas and indices, e.g. G\&K, RI and KF2, performed well on our dataset.

\section{Evaluation of the proposed discriminant model}

To develop a machine learning-based discriminating model, prediction performance depends on the parameter(s) used. In this study, five popular classifiers were considered, e.g. $k$-NN, DT, RF, ANN and SVM. To make fair comparison with existing formulas and indices, the same dataset was used. Furthermore, a 5-fold CV and external validation tests were carried out. As described above, the internal and external datasets were constructed with a random sampling procedure. To objectively evaluate the impact of the random sampling procedure, we repeated this construction 100 times. Table 4 and Fig. 4 list performance comparisons of various models using different methods over the 5-fold CV and external validation schemes.

As seen in Table 4, the DT model yielded the highest prediction results over the 5 -fold $\mathrm{CV}$ with a mean Ac, Sp, Sn, MCC, YI, and AUC of 98.03, 96.15, 98.54\%, 0.94, 0.93 , and 1.00, respectively, while the SVM and RF models performed well with the second and third best prediction results. On the other hand, the SVM model

Table 2 The age and red blood cell parameters of study subjects with thalassemia trait or iron deficiency anemia

\begin{tabular}{llll}
\hline Parameters & $T(N=146)$ & IDA $(N=40)$ & $p$-value \\
\hline Age (yrs.) & $37.79 \pm 7.86(18.00-50.00)$ & $39.15 \pm 9.61(23.00-58.00)$ & 0.435 \\
RBC $\left(10^{6} / \mu \mathrm{L}\right)$ & $5.32 \pm 0.48(4.35-6.83)$ & $4.03 \pm 0.96(1.69-5.77)$ & $<0.001^{*}$ \\
Hb (g/dL) & $11.99 \pm 1.11(8.10-14.60)$ & $7.97 \pm 2.26(2.50-10.90)$ & $<0.001^{*}$ \\
Hct (\%) & $36.46 \pm 4.10(34.10-42.40)$ & $26.19 \pm 6.63(9.90-34.30)$ & $<0.001^{*}$ \\
MCV (fL) & $69.49 \pm 6.14(52.30-79.70)$ & $65.12 \pm 9.32(48.70-81.00)$ & $0.010^{*}$ \\
MCH (pg) & $22.71 \pm 2.25(17.70-26.60)$ & $19.65 \pm 3.33(12.50-25.70)$ & $<0.001^{*}$ \\
MCHC (\%) & $32.67 \pm 1.14(30.10-35.70)$ & $30.13 \pm 1.93(24.90-35.70)$ & $<0.001^{*}$ \\
RDW (\%) & $15.88 \pm 1.13(13.50-22.00)$ & $20.48 \pm 3.23(14.90-26.70)$ & $<0.001^{*}$ \\
\hline
\end{tabular}

The data are shown as mean \pm standard deviation

$\mathrm{Hb}$ Hemoglobin; Hct Hematocrit; IDA Iron deficiency anemia; $M C H$ mean corpuscular hemoglobin; $M C H C$ mean corpuscular hemoglobin concentration; $M C V$ mean corpuscular volume; $R B C$ red blood cell count; $R D W$ red blood cell distribution width; $\pi$ Thalassemia trait

* Mann-Whitney $U$ test $p$-value $<0.05$ 

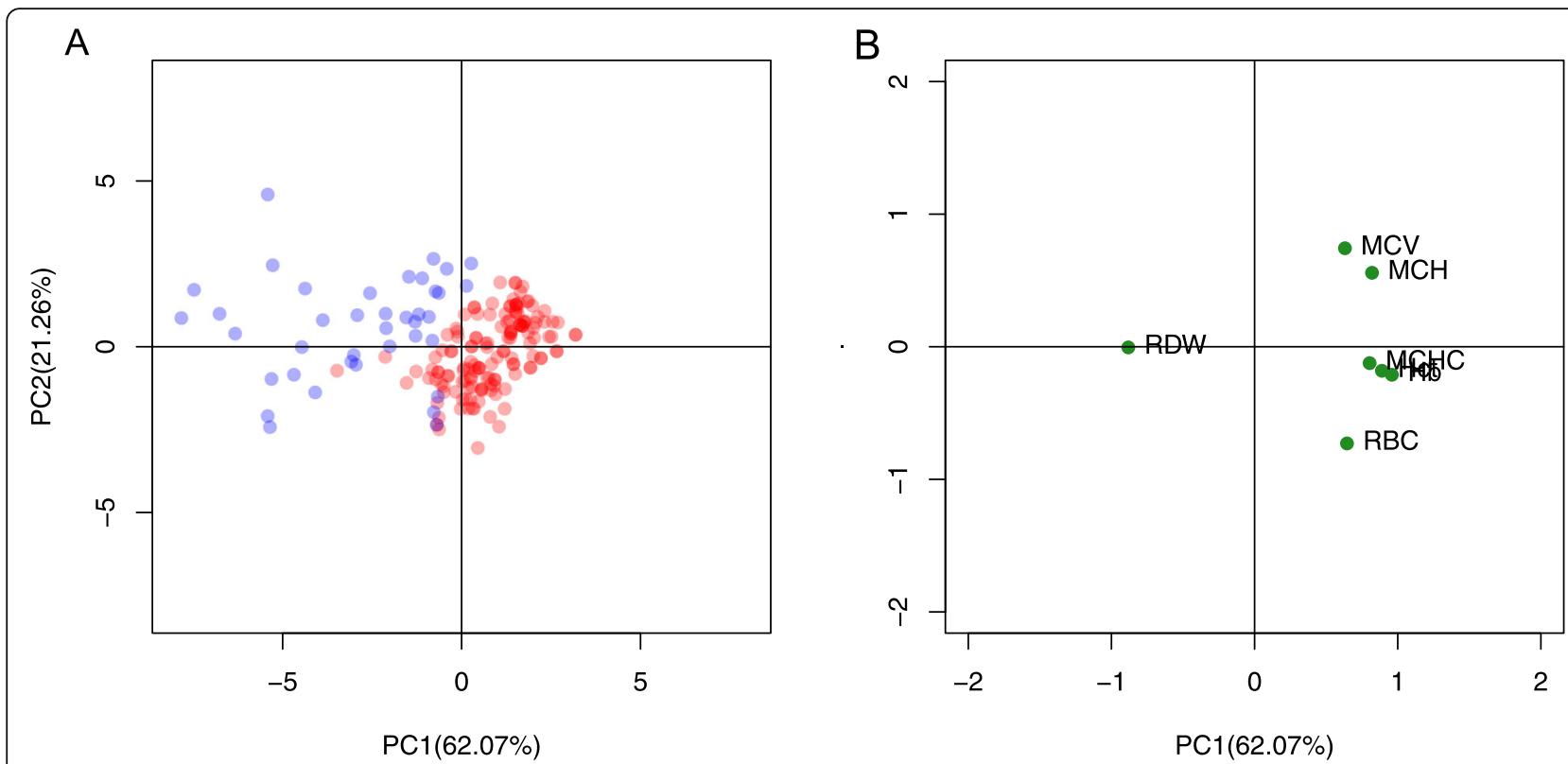

Fig. 2 Multivariate analysis using principal component analysis (PCA) of our laboratory data consisting of 146 TT cases (red circles) and 40 IDA cases (blue circles) derived from PCA scores (a) and loadings (b) plots

was more effective than the DT model over the external validation test with a mean Ac, Sp, Sn, MCC, YI, and AUC of 95.59, 92. 49\%, 96.74\%, 0.87, 0.87, and 0.98, respectively. Interestingly, the SVM model still achieved levels of discrimination between IDA and TT on the independent dataset with means of Ac, Sp, Sn, MCC, YI, and AUC being 96.08, 92.59, 100.00\%, 0.92, 0.92, and 1.00 , respectively. As mentioned in the section Data collection, the dataset used in this study is imbalance among IDA and TT samples. Thus, we utilized a resampling approach to randomly generate a balanced internal set consisting of 32 IDA and 32 TT samples, the remaining samples consisting of 8 IDA and 114 TT sample were used as an external set. To objectively evaluate the impact of random sampling, we repeated this process with 100 independent iterations to generate the balanced internal and new external sets for constructing SVM models. The average prediction results (Ac and MCC)

Table 3 Performance comparisons of existing discriminant formulas and indices proposed for differentiation of iron deficiency anemia from thalassemia trait

\begin{tabular}{|c|c|c|c|c|c|c|c|}
\hline Indices/ formulas & Cut-off & $A c(\%)$ & Sn (\%) & Sp (\%) & MCC & $\mathrm{Yl}$ & $A \cup C$ \\
\hline $\mathrm{Bl}=\mathrm{RDW}$ & 15 & 36.02 & 19.18 & 97.50 & 0.19 & 0.17 & 0.71 \\
\hline$E F=M C V-10 \times R B C$ & 15 & 52.15 & 45.89 & 75.00 & 0.17 & 0.21 & 0.70 \\
\hline$E \& F=M C V-R B C-5 H b-6.4$ & 0 & 67.42 & 60.95 & 92.50 & 0.44 & 0.54 & 0.91 \\
\hline $\mathrm{G} \& \mathrm{~K}=\mathrm{MCV} \mathrm{V}^{2} \times \mathrm{RDW} / 100 \mathrm{Hb}$ & 72 & 84.95 & 82.88 & 92.50 & 0.66 & 0.75 & 0.93 \\
\hline $\mathrm{Ml}=\mathrm{MCV} / \mathrm{RBC}$ & 13 & 54.30 & 47.95 & 77.50 & 0.21 & 0.25 & 0.75 \\
\hline $\mathrm{RDWI}=\mathrm{MCV} \times \mathrm{RDW} / \mathrm{RBC}$ & 220 & 67.20 & 60.96 & 90.00 & 0.42 & 0.51 & 0.92 \\
\hline $\mathrm{Rl}=\mathrm{RDW} / \mathrm{RBC}$ & 3.3 & 87.63 & 86.30 & 92.50 & 0.70 & 0.79 & 0.98 \\
\hline$S \& L=M C V^{2} \times M C H / 100$ & 1530 & 74.19 & 92.47 & 7.50 & -0.01 & 0.00 & 0.31 \\
\hline $\mathrm{SI}=\mathrm{MCV}-\mathrm{RBC}-3 \mathrm{Hb}$ & 27 & 53.76 & 45.21 & 85.00 & 0.26 & 0.302 & 0.80 \\
\hline $\mathrm{SF}=\mathrm{MCH} / \mathrm{RBC}$ & 3.8 & 37.63 & 26.71 & 77.50 & 0.04 & 0.04 & 0.68 \\
\hline $\mathrm{SiF}=1.5 \mathrm{Hb}-0.05 \mathrm{MCV}$ & 14 & 24.73 & 31.51 & 0.00 & -0.56 & -0.69 & 0.02 \\
\hline $\mathrm{KF} 1=\mathrm{RBC} / \mathrm{Hct}+0.5 \mathrm{RDW}$ & 8.2 & 70.97 & 64.38 & 95.00 & 0.49 & 0.59 & 0.93 \\
\hline $\mathrm{KF} 2=5 \mathrm{RDW} / \mathrm{RBC}$ & 16.8 & 89.79 & 89.04 & 92.50 & 0.74 & 0.82 & 0.98 \\
\hline
\end{tabular}

Ac Accuracy; AUC Area under receiver operating curve; Hb Hemoglobin; Hct Hematocrit; IDA Iron deficiency anemia; $M C C$ Matthew's correlation coefficient; $M C H$ mean corpuscular hemoglobin; $M C V$ mean corpuscular volume; $R B C$ red blood cell count; RDW red blood cell distribution width; Sn Sensitivity; Sp Specificity; $\pi$ Thalassemia trait; $Y I$ Youden's index 




False positive rate

Fig. 3 Performance comparison of existing discriminant formulas and indices using ROC curves

Table 4 Performance comparisons between DT, RF and SVM models in differentiation of iron deficiency anemia from thalassemia trait

\begin{tabular}{|c|c|c|c|c|c|c|c|}
\hline Classifier & Validation & $A c(\%)$ & $\mathrm{Sp}(\%)$ & Sn (\%) & MCC & $\mathrm{YI}$ & $A \cup C$ \\
\hline \multirow[t]{3}{*}{ k-NN } & 5-fold CV & $92.36 \pm 1.67$ & $90.48 \pm 3.62$ & $92.80 \pm 1.76$ & $0.76 \pm 0.06$ & $0.83 \pm 0.04$ & $0.81 \pm 0.07$ \\
\hline & External & $92.54 \pm 4.26$ & $90.09 \pm 10.79$ & $93.35 \pm 4.22$ & $0.77 \pm 0.14$ & $0.83 \pm 0.13$ & $0.80 \pm 0.10$ \\
\hline & Independent & 90.20 & 83.33 & 100.00 & 0.82 & 0.83 & 0.85 \\
\hline \multirow[t]{3}{*}{ DT } & 5-fold CV & $98.03 \pm 0.91$ & $96.15 \pm 3.40$ & $98.54 \pm 0.99$ & $0.94 \pm 0.026$ & $0.93 \pm 0.06$ & $1.00 \pm 0.00$ \\
\hline & External & $93.83 \pm 4.10$ & $86.86 \pm 11.16$ & $96.22 \pm 3.77$ & $0.82 \pm 0.12$ & $0.82 \pm 0.04$ & $0.92 \pm 0.06$ \\
\hline & Independent & 92.16 & 86.21 & 100.00 & 0.85 & 0.85 & 1.00 \\
\hline \multirow[t]{3}{*}{ RF } & 5-fold CV & $94.17 \pm 1.26$ & $88.15 \pm 3.49$ & $95.75 \pm 0.95$ & $0.83 \pm 0.04$ & $0.83 \pm 0.04$ & $0.97 \pm 0.01$ \\
\hline & External & $94.62 \pm 3.29$ & $90.07 \pm 8.99$ & $96.13 \pm 3.19$ & $0.84 \pm 0.10$ & $0.84 \pm 0.07$ & $0.98 \pm 0.02$ \\
\hline & Independent & 92.16 & 86.21 & 100.00 & 0.85 & 0.85 & 1.00 \\
\hline \multirow[t]{3}{*}{ ANN } & 5-fold CV & $94.11 \pm 1.31$ & $86.75 \pm 3.58$ & $96.14 \pm 1.06$ & $0.83 \pm 0.04$ & $0.83 \pm 0.04$ & $0.97 \pm 0.02$ \\
\hline & External & $93.78 \pm 3.71$ & $86.81 \pm 10.84$ & $96.22 \pm 3.30$ & $0.82 \pm 0.11$ & $0.83 \pm 0.11$ & $0.98 \pm 0.02$ \\
\hline & Independent & 94.12 & 89.29 & 100.00 & 0.89 & 0.89 & 1.00 \\
\hline \multirow[t]{3}{*}{ SVM } & 5-fold CV & $95.05 \pm 1.06$ & $89.81 \pm 2.66$ & $96.45 \pm 0.96$ & $0.85 \pm 0.03$ & $0.85 \pm 0.04$ & $0.97 \pm 0.01$ \\
\hline & External & $95.59 \pm 2.76$ & $92.49 \pm 8.47$ & $96.74 \pm 2.59$ & $0.87 \pm 0.08$ & $0.87 \pm 0.10$ & $0.98 \pm 0.03$ \\
\hline & Independent & 96.08 & 92.59 & 100.00 & 0.92 & 0.92 & 1.00 \\
\hline
\end{tabular}

The data are shown as mean \pm standard deviation (100 times)

Ac Accuracy; ANN Artificial neural network; AUC Area under receiver operating curve; DT Decision tree; $k$-NN k-nearest neighbor; MCC Matthew's correlation coefficient; RF Random forest; Sn Sensitivity; Sp Specificity; SVM Support vector machine; YI Youden's index; 5-fold CV 5-fold cross validation

Parameters of $k$-NN $(k)$, RF (ntree, mtry), ANN (size, decay) and SVM (cost, $\gamma$ ) were optimized by a 5-fold CV procedure. Values of $k$, ntree, mtry, size, decay, cost and $\gamma$ are $5,200,2,4,0.5,8$ and 0.5 

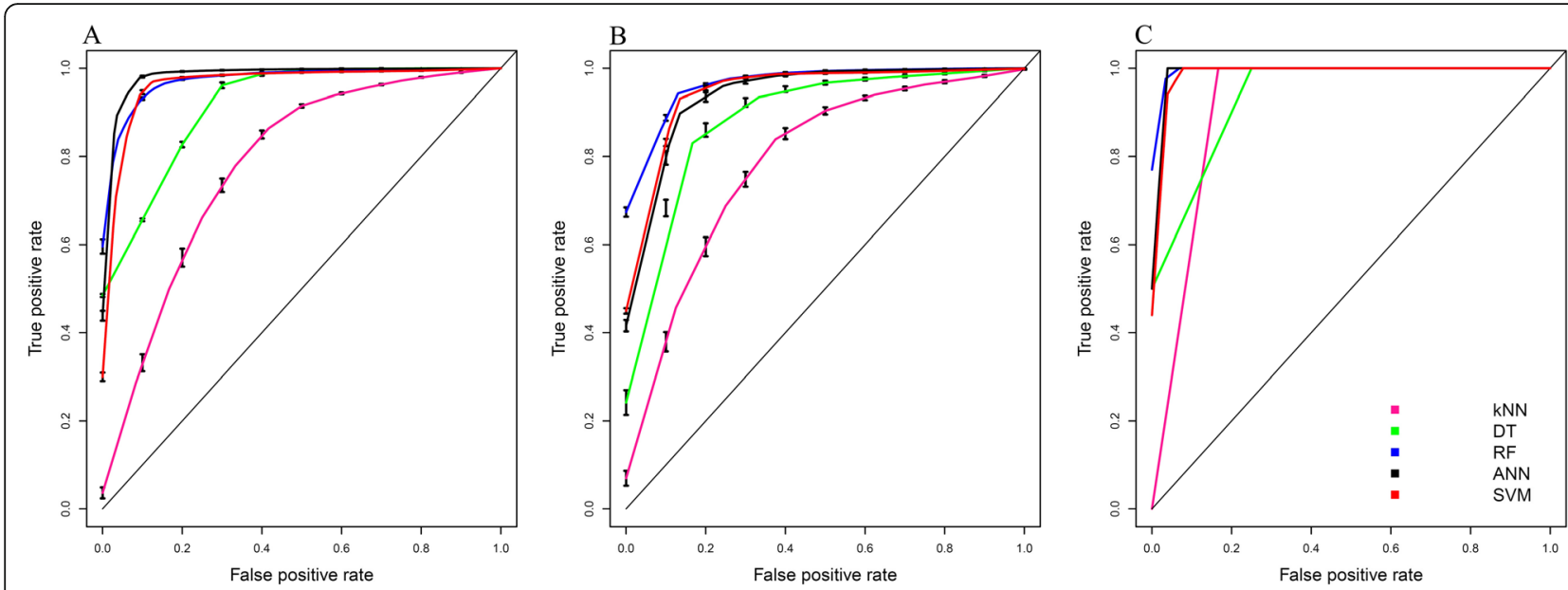

Fig. 4 Performance comparisons among k-NN, DT, RF, ANN and SVM models using ROC curves over 5-fold CV (a) external test (b) and independent test (c), where k-NN, DT, RF, ANN and SVM models are represented by pink, green, red, black and blue, respectively

performed on the balanced internal and new external sets were $(92.75 \%, 0.88)$ and $(94.30 \%, 0.77)$, respectively. These results indicated that SVM model can tackle the imbalanced dataset problem and provide desirable prediction results [48].

By observing the performance comparisons in Table 4 and Fig. 4, we conclude that DT model shows the highest performance level when evaluated by 5 -fold $\mathrm{CV}$, while SVM model outperform that other conventional models over the external validation test and independent dataset. This observation is consistent with the previous works $[28,49]$. Furthermore, many studies have mentioned that the overfitting is the major problem of DT model [50,51]. For convenience, this best predictor for discriminating between IDA and TT (based on SVM model) will be referred to as 'ThalPred'.

For convenience, this best predictor for discriminating between IDA and TT (based on SVM model) will be referred to as 'ThalPred'.

\section{Extracted important rules obtained from the RF model}

The interpretable rules were established by using the RF model to demonstrate the combination of RBC indices for discriminating IDA from TT. Table 5 presents the eight interpretable rules as conditions in a simple linguistic manner, where Frequency (\%) is the percentage of a data satisfying a condition, Error is the error percentage of a rule and Prediction is the outcome of a rule.

The first rule, which covers $63.98 \%$ of the whole data, was constructed with single RBC indices, e.g. Hb. This rule has one criterion: if the value of $\mathrm{Hb}$ is larger than $10.95 \mathrm{~g} / \mathrm{dL}$, then the prediction is TT. The second rule, which covers $15.05 \%$ of whole data, was constructed with two RBC indices, e.g. $\mathrm{RBC}$ and $\mathrm{Hb}$. This rule has two criteria: if (i) the value of $\mathrm{RBC}$ is equal to or less than $4.5 \times 10^{6} / \mu \mathrm{L}$ and (ii) the value of $\mathrm{Hb}$ is equal to or less than $10.45 \mathrm{~g} / \mathrm{dL}$, then the prediction is IDA. The third rule, which covers $11.19 \%$ of the whole data, was depicted with single RBC indices, e.g. RDW. This rule has one criterion: if the value of RDW is equal or less than $17.15 \%$, then the prediction is TT. The fourth rule, which covers $4.84 \%$ of the whole data, is constructed with three $\mathrm{RBC}$ indices, e.g. $\mathrm{RBC}, \mathrm{Hb}$ and $\mathrm{RDW}$. This rule has three criteria: if (i) the value of $\mathrm{RBC}$ is greater than $4.59 \times 10^{6} / \mu \mathrm{L}$ and (ii) the value of $\mathrm{Hb}$ is equal to or

Table 5 The extracted interpretable rules derived from RF model in differentiation of iron deficiency anemia from thalassemia trait

\begin{tabular}{lllll}
\hline Length & Frequency (\%) & Error (\%) & Condition & Prediction \\
\hline 1 & 63.98 & 0.00 & $\mathrm{Hb}>10.95$ & $T$ \\
2 & 15.05 & 0.00 & $\mathrm{RBC} \leq 4.5$ and $\mathrm{Hb} \leq 10.45$ & IDA \\
1 & 11.29 & 0.00 & $\mathrm{RDW} \leq 17.15$ & $\Pi$ \\
3 & 4.84 & 0.00 & $\mathrm{RBC}>4.59$ and $\mathrm{Hb} \leq 10.95$ and RDW $>17.7$ & IDA \\
4 & 2.15 & 0.00 & $\mathrm{RBC}>4.28$ and $\mathrm{MCHC} \leq 32.15$ and RDW $>17.15$ and RDW $\leq 17.7$ & $\Pi$ \\
3 & 1.08 & 0.00 & $\mathrm{Hb} \leq 11.45$ and $\mathrm{MCHC}>31.35$ and RDW $>17.4$ & IDA \\
1 & 1.61 & 33.00 & Else & $\Pi$ \\
\hline
\end{tabular}


less than $10.95 \mathrm{~g} / \mathrm{dL}$ and (iii) the value of RDW is greater than $17.7 \%$, then the prediction is IDA. The fifth rule, which covers $2.15 \%$ of whole data, was constructed with three $\mathrm{RBC}$ indices, e.g. $\mathrm{RBC}, \mathrm{MCHC}$ and $\mathrm{RDW}$. This rule has four criteria: if (i) the value of $\mathrm{RBC}$ is greater than $4.28 \times 10^{6} / \mu \mathrm{L}$ and (ii) the value of $\mathrm{MCHC}$ is equal to or less than $32.15 \mathrm{~g} / \mathrm{dL}$ and (iii) the value of RDW is greater than $17.15 \%$ and (iv) the value of RDW is greater than $17.7 \%$, then the prediction is TT. The sixth rule, which covers $1.08 \%$ of whole data, was constructed with three RBC indices, e.g. $\mathrm{Hb}, \mathrm{MCHC}$ and RDW. This rule has three criteria: if (i) the value of $\mathrm{Hb}$ is equal to or less than $11.45 \mathrm{~g} / \mathrm{dL}$, (ii) the value of $\mathrm{MCHC}$ is greater than $31.35 \mathrm{~g} / \mathrm{dL}$, and (iii) the value of RDW is greater than $17.4 \%$, then the prediction is IDA. The seventh rule covers $1.61 \%$ of the whole data. If a query has RBC indices which do not satisfy any of the seven interpretable rules, then it is classified as TT with an error of $33.00 \%$.

\section{Web-based tool implementation}

For the convenience of health care team, based on the best model (e.g. SVM model) proposed in the present work, a publicly accessible web-based tool for ThalPred has been established. A screenshot of the ThalPred is shown in Fig. 5. Furthermore, to maximize user's convenience, a step-by-step walkthrough of the procedures for using the ThalPred web based tool is provided below.

Step 1. Open the web-based tool at http://codes.bio/ thalpred/.
Step 2. There are two options for prediction:

1) Key the single data in the input parameters panel.

2) Upload the query patient's $R B C$ indices $(R B C, H b$, Hct, $\mathrm{MCV}, \mathrm{MCH}, \mathrm{MCHC}$ and $\mathrm{RDW}$ ) into the input box by clicking on the Choose file button. The input data should be in CSV format. For example, of patient's RBC indices in CSV format, click the insert example data above the input box. Finally, press on the Submit button to initiate the prediction process.

Step 3. Prediction results are automatically displayed in a grey box found below the Status/Output heading. Users can also download the prediction results as a CSV file by pressing on the Download results bottom.

\section{Discussion}

Anemia is crucial problem occurring with population in Thailand and affecting to health and economic system. HMA, such as TT and IDA, are found commonly in clinical laboratory. Discrimination between TT and IDA group is essential for correct genetic counseling and effective treatment. $\mathrm{RBC}$ indices, consisting of $\mathrm{RBC}, \mathrm{Hb}$, $\mathrm{Hct}, \mathrm{MCV}, \mathrm{MCH}, \mathrm{MCHC}$ and $\mathrm{RDW}$, are generated from complete blood count $(\mathrm{CBC})$ analysis using automated instruments. Therefore, using parameters from the $\mathrm{CBC}$ result for differentiation is of much interest. Generally, the $\mathrm{RBC}$ count, $\mathrm{Hb}, \mathrm{MCV}, \mathrm{MCH}$ and $\mathrm{RDW}$ are used to formulate a new index. As a result of the anemic condition, subjects with TT are found to have an increased $\mathrm{RBC}$ count $[22,52,53]$. While blood films from individuals with TT or IDA have shown different levels of

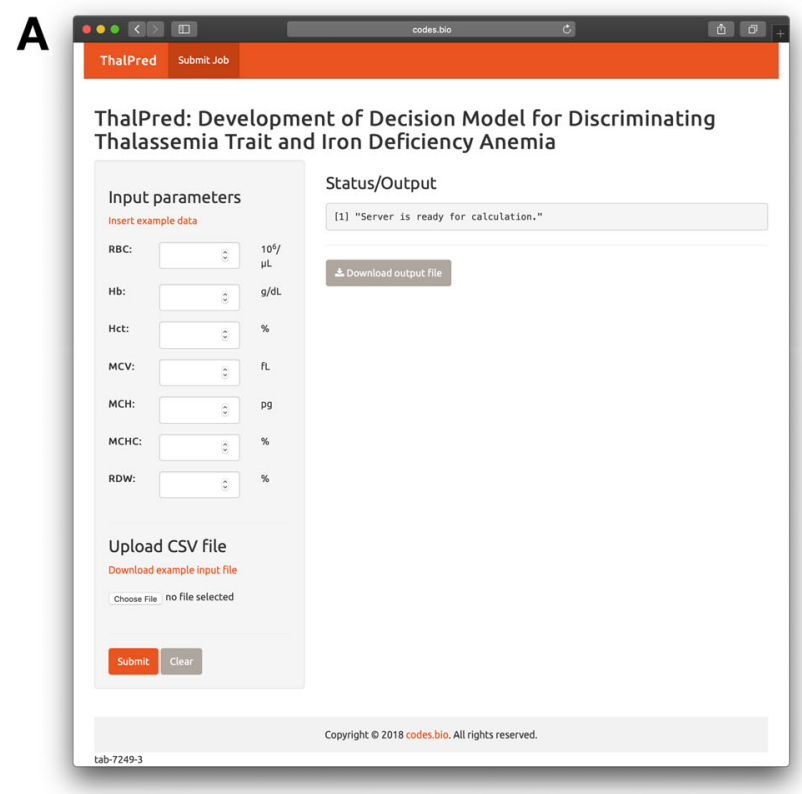

B

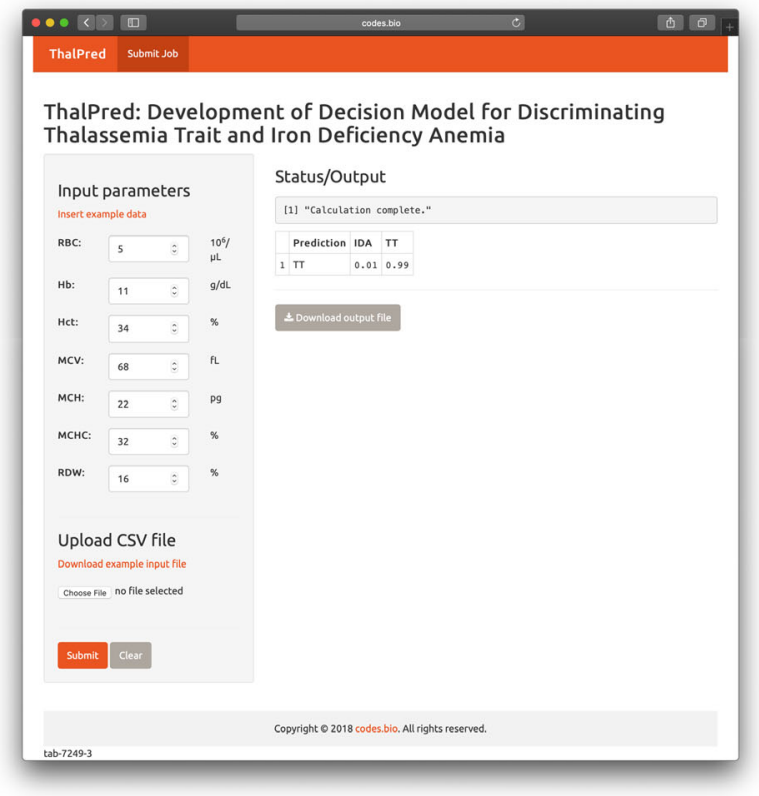

Fig. 5 Screenshots of the ThalPred web-based tool before (a) and after (b, c) submission of laboratory data, which is available at http://codes.bio/thalpred/ 
Table 6 The prediction results derived from DT, RF and SVM models in differentiation of iron deficiency anemia (IDA) from thalassemia trait (TT)

\begin{tabular}{|c|c|c|c|c|c|c|c|c|c|c|c|c|c|}
\hline \multirow[t]{2}{*}{ No. } & \multirow[t]{2}{*}{$\mathrm{RBC}$} & \multirow[t]{2}{*}{$\mathrm{Hb}$} & \multirow[t]{2}{*}{$\mathrm{Hct}$} & \multirow[t]{2}{*}{ MCV } & \multirow[t]{2}{*}{$\mathrm{MCH}$} & \multirow[t]{2}{*}{$\mathrm{MCHC}$} & \multirow[t]{2}{*}{ RDW } & \multirow[t]{2}{*}{ Diagnosis } & \multicolumn{5}{|c|}{ Prediction } \\
\hline & & & & & & & & & $k-N N$ & DT & RF & ANN & SVM \\
\hline 1 & 5.35 & 10.6 & 33.0 & 61.7 & 19.8 & 32.1 & 13.7 & $\pi$ & $\pi$ & $\pi$ & $\pi$ & $\pi$ & $\pi$ \\
\hline 2 & 5.40 & 10.9 & 34.1 & 63.2 & 20.2 & 32.0 & 14.5 & $\pi$ & $\pi$ & $\pi$ & $\pi$ & $\pi$ & $\pi$ \\
\hline 3 & 5.40 & 10.3 & 33.0 & 61.1 & 19.1 & 31.2 & 14.2 & $\Pi$ & $\pi$ & $\pi$ & $\pi$ & $\pi$ & $\pi$ \\
\hline 4 & 6.01 & 12.3 & 38.3 & 63.7 & 20.5 & 32.1 & 13.2 & $\Pi T$ & $\pi$ & $\pi$ & $\pi$ & $\pi$ & $\pi$ \\
\hline 5 & 5.55 & 11.0 & 33.4 & 60.2 & 19.8 & 32.9 & 13.2 & $\Pi$ & $\pi$ & $\pi$ & $\pi$ & $\Pi$ & $\pi$ \\
\hline 6 & 6.04 & 12.9 & 39.6 & 65.6 & 21.4 & 32.6 & 13.5 & $\pi$ & $\pi$ & $\pi$ & $\pi$ & $\pi$ & $\pi$ \\
\hline 7 & 5.90 & 13.1 & 40.0 & 67.8 & 22.2 & 32.8 & 12.7 & $\pi$ & $\pi$ & $\pi$ & $\pi$ & $\pi$ & $\pi$ \\
\hline 8 & 5.95 & 12.6 & 39.0 & 65.6 & 21.2 & 32.3 & 13.4 & $\pi$ & $\pi$ & $\pi$ & $\pi$ & $\pi$ & $\pi$ \\
\hline 9 & 6.11 & 12.7 & 39.5 & 64.7 & 20.8 & 32.2 & 13.7 & $\pi$ & $\pi$ & $\pi$ & $\pi$ & $\pi$ & $\pi$ \\
\hline 10 & 5.45 & 11.8 & 36.6 & 67.1 & 21.7 & 32.3 & 14.0 & $\pi$ & $\pi$ & $\pi$ & $\pi$ & $\pi$ & $\pi$ \\
\hline 11 & 5.40 & 11.0 & 34.0 & 63.0 & 20.4 & 32.4 & 12.8 & $\pi$ & $\pi$ & $\Pi$ & $\pi$ & $\pi$ & $\pi$ \\
\hline 12 & 5.40 & 11.0 & 34.0 & 63.0 & 20.4 & 32.4 & 14.0 & $\pi$ & $\pi$ & $\pi$ & $\pi$ & $\pi$ & $\pi$ \\
\hline 13 & 6.20 & 12.4 & 37.2 & 60.0 & 20.0 & 33.3 & 12.6 & $\pi$ & $\Pi$ & $\pi$ & $\pi$ & $\pi$ & $\pi$ \\
\hline 14 & 5.40 & 10.7 & 33.0 & 61.1 & 19.8 & 32.4 & 13.6 & $\pi$ & $\pi$ & $\pi$ & $\pi$ & $\pi$ & $\pi$ \\
\hline 15 & 6.11 & 12.3 & 38.0 & 62.2 & 20.1 & 32.4 & 12.6 & $\Pi$ & $\pi$ & $\pi$ & $\pi$ & $\pi$ & $\pi$ \\
\hline 16 & 3.40 & 7.7 & 24.1 & 70.9 & 22.7 & 32.0 & 20.1 & IDA & IDA & IDA & IDA & IDA & IDA \\
\hline 17 & 4.66 & 11.3 & 34.4 & 73.8 & 24.3 & 32.9 & 21.0 & IDA & $\pi$ & $\pi$ & $\pi$ & IDA & $\pi$ \\
\hline 18 & 4.54 & 10.6 & 32.8 & 72.3 & 23.4 & 32.3 & 21.0 & IDA & IDA & IDA & IDA & IDA & IDA \\
\hline 19 & 3.50 & 7.9 & 25.2 & 72.0 & 22.6 & 31.4 & 21.2 & IDA & IDA & IDA & IDA & IDA & IDA \\
\hline 20 & 4.15 & 9.9 & 29.0 & 69.9 & 23.9 & 34.1 & 20.2 & IDA & $\pi$ & IDA & IDA & IDA & IDA \\
\hline 21 & 3.90 & 8.9 & 28.0 & 71.8 & 22.8 & 31.8 & 20.2 & IDA & IDA & IDA & IDA & IDA & IDA \\
\hline 22 & 4.17 & 9.9 & 29.0 & 69.5 & 23.7 & 34.1 & 21.1 & IDA & IDA & IDA & IDA & IDA & IDA \\
\hline 23 & 3.85 & 8.5 & 27.5 & 71.4 & 22.1 & 30.9 & 21.3 & IDA & IDA & IDA & IDA & IDA & IDA \\
\hline 24 & 4.24 & 9.8 & 30.5 & 71.9 & 23.1 & 32.1 & 20.2 & IDA & IDA & IDA & IDA & IDA & IDA \\
\hline 25 & 4.83 & 11.1 & 34.0 & 70.4 & 23.0 & 32.7 & 19.0 & IDA & $\pi$ & $\pi$ & $\pi$ & $\pi$ & $\pi$ \\
\hline 26 & 4.64 & 10.6 & 33.0 & 71.1 & 22.8 & 32.1 & 20.5 & IDA & IDA & IDA & IDA & IDA & $\mathrm{IDA}$ \\
\hline 27 & 4.01 & 9.5 & 29.0 & 72.3 & 23.7 & 32.8 & 20.4 & IDA & IDA & IDA & IDA & IDA & $\mathrm{IDA}$ \\
\hline 28 & 4.80 & 11.0 & 34.0 & 70.8 & 22.9 & 32.4 & 21.1 & IDA & $\pi$ & $\pi$ & $\pi$ & IDA & IDA \\
\hline 29 & 3.65 & 8.4 & 26.0 & 71.2 & 23.0 & 32.3 & 20.4 & IDA & IDA & IDA & IDA & IDA & $\mathrm{IDA}$ \\
\hline 30 & 4.00 & 9.2 & 28.0 & 70.0 & 23.0 & 32.9 & 20.1 & IDA & IDA & IDA & IDA & IDA & IDA \\
\hline 31 & 4.45 & 10.2 & 31.4 & 70.6 & 22.9 & 32.5 & 19.8 & IDA & IDA & IDA & IDA & IDA & $\mathrm{IDA}$ \\
\hline 32 & 4.44 & 10.3 & 32.0 & 72.1 & 23.2 & 32.2 & 21.3 & IDA & IDA & IDA & IDA & IDA & $\mathrm{IDA}$ \\
\hline 33 & 4.56 & 10.4 & 32.0 & 70.2 & 22.8 & 32.5 & 19.4 & IDA & IDA & IDA & IDA & IDA & $\mathrm{IDA}$ \\
\hline 34 & 4.84 & 11.0 & 34.0 & 70.3 & 22.7 & 32.4 & 20.7 & IDA & $\pi$ & $\pi$ & $\pi$ & IDA & IDA \\
\hline 36 & 3.83 & 8.7 & 27.0 & 70.5 & 22.72 & 32.22 & 20.0 & IDA & IDA & IDA & IDA & IDA & IDA \\
\hline 37 & 3.6 & 8.7 & 25.0 & 69.44 & 24.17 & 34.8 & 19.3 & IDA & IDA & IDA & IDA & IDA & IDA \\
\hline 38 & 3.52 & 8.0 & 25.0 & 71.02 & 22.73 & 32 & 21.1 & IDA & IDA & IDA & IDA & IDA & IDA \\
\hline 39 & 3.98 & 9.0 & 27.8 & 69.85 & 22.61 & 32.37 & 20.7 & IDA & IDA & IDA & IDA & IDA & IDA \\
\hline 40 & 4.02 & 9.0 & 28.5 & 70.9 & 22.39 & 31.58 & 21.1 & IDA & IDA & IDA & IDA & IDA & IDA \\
\hline 41 & 4.43 & 10.1 & 31.4 & 70.88 & 22.8 & 32.17 & 19.8 & IDA & IDA & IDA & IDA & IDA & IDA \\
\hline 42 & 4.24 & 9.6 & 30.0 & 70.75 & 22.64 & 32.0 & 21.0 & $\pi$ & $\pi$ & $\pi$ & $\pi$ & $\pi$ & $\pi$ \\
\hline 43 & 5.94 & 12.6 & 39.0 & 65.7 & 21.2 & 32.3 & 13.0 & $\Pi$ & IDA & $\pi$ & $\pi$ & IDA & $\Pi$ \\
\hline
\end{tabular}


Table 6 The prediction results derived from DT, RF and SVM models in differentiation of iron deficiency anemia (IDA) from thalassemia trait (TT) (Continued)

\begin{tabular}{|c|c|c|c|c|c|c|c|c|c|c|c|c|c|}
\hline \multirow[t]{2}{*}{ No. } & \multirow[t]{2}{*}{$\mathrm{RBC}$} & \multirow[t]{2}{*}{$\mathrm{Hb}$} & \multirow[t]{2}{*}{$\mathrm{Hct}$} & \multirow[t]{2}{*}{$\mathrm{MCV}$} & \multirow[t]{2}{*}{$\mathrm{MCH}$} & \multirow[t]{2}{*}{$\mathrm{MCHC}$} & \multirow[t]{2}{*}{ RDW } & \multirow[t]{2}{*}{ Diagnosis } & \multicolumn{5}{|c|}{ Prediction } \\
\hline & & & & & & & & & $k$-NN & DT & RF & ANN & SVM \\
\hline$\overline{44}$ & 5.80 & 11.7 & 36.3 & 62.6 & 20.2 & 32.2 & 13.1 & $\pi$ & IDA & $\pi$ & $\pi$ & IDA & $\pi$ \\
\hline 45 & 5.45 & 11.4 & 35.0 & 64.2 & 20.9 & 32.6 & 13.0 & $\pi$ & IDA & $\pi$ & $\pi$ & IDA & $\pi$ \\
\hline 46 & 5.40 & 11.0 & 33.7 & 62.4 & 20.4 & 32.6 & 12.5 & $\pi$ & IDA & $\pi$ & $\pi$ & IDA & $\pi$ \\
\hline 47 & 6.11 & 12.6 & 38.5 & 63.0 & 20.6 & 32.7 & 14.3 & $\pi$ & IDA & $\pi$ & $\pi$ & IDA & $\pi$ \\
\hline 48 & 5.80 & 12.2 & 36.4 & 62.8 & 21.0 & 33.5 & 14.0 & $\pi$ & IDA & $\pi$ & $\pi$ & IDA & $\pi$ \\
\hline 49 & 5.44 & 10.8 & 34.0 & 62.5 & 19.9 & 31.8 & 12.7 & $\pi$ & IDA & $\pi$ & $\pi$ & IDA & $\pi$ \\
\hline 50 & 5.40 & 10.8 & 33.0 & 61.1 & 20.0 & 32.7 & 13.6 & $\pi$ & $\pi$ & $\pi$ & $\pi$ & $\pi$ & $\pi$ \\
\hline 51 & 6.05 & 12.3 & 38.0 & 62.8 & 20.3 & 32.4 & 14.0 & $\pi$ & $\pi$ & $\pi$ & $\pi$ & $\pi$ & $\pi$ \\
\hline
\end{tabular}

ANN Artificial neural network; DT Decision tree; Hb Hemoglobin; Hct Hematocrit; IDA Iron deficiency anemia; $k$-NN $k$-nearest neighbor; $M C H$ mean corpuscular hemoglobin; $M C H C$ mean corpuscular hemoglobin concentration; $M C V$ mean corpuscular volume; RBC red blood cell count; RDW red blood cell distribution width; RF Random forest; SVM Support vector machine; $\pi$ Thalassemia trait. Parameters of $k$-NN ( $k$ ), RF (ntree, mtry), ANN (size, decay) and SVM (cost, $\gamma$ ) were optimized by a 5 -fold CV procedure. Values of $k$, ntree, mtry, size, decay, cost and $\gamma$ are $5,200,2,4,0.5,8$ and 0.5

anisocytosis (a condition of variation in sizes of RBC). Most of IDA patients appeared to have higher RDW than TT patients $[22,54]$. The results of both univariate (Table 2) and multivariate (Fig. 2) analyses are agreeable with previous studies. Hence, one parameter is not enough to discriminate between these two conditions and all RBC parameters have potential role on the differentiation.

Previously, several discriminant formulas and indices have been proposed by different researchers for discrimination between IDA from TT [8-19]. However, these formulas and indices are not appropriate for every population. In Thailand, many researchers attempted to utilize these existing formulas and indices with different interest groups, e.g. school children [55], adults [56], anemic vegetarians [57], etc. In this study, the group of dataset composed of adults between 18 to 50 years old. Our finding showed that G\&K, RI and KF2 performed well on our dataset. Interestingly, the top- three formulas and indices demonstrated the highest performance harboring of RDW and 2 of 3 consisting of RBC parameter.

Prior studies utilizing data from Thai people also found that most formulas containing $\mathrm{RBC}$ and RDW yielded a high performance. Pornprasert et al. suggested that SI and SF are the most reliable formulas [55], whereas RI was found to be most efficient in studies by Plengsuree et al. [56] and Sirachainan et al [18]. Conversely, S\&L (which does not contain RBC and RDW) was proposed as a suitable index for differentiation in young Asians. It achieved 100\% Sn and high Sp in Indian and Taiwanese $[22,54]$. Our findings demonstrated consistent results to the previous publications that $\mathrm{RBC}$ and RDW play key roles in accurately distinguishing these two diseases. However, $89.8 \%$ Ac is not sufficient for medical decision making $[58,59]$. Therefore, we attempted to increase the efficiency and accuracy of discrimination between TT and IDA in Thai adults by constructing a new model from hematological indices via machine learning approach.

As seen in Table 3 and Fig. 3, the prediction performances of the existing formulas and indices are still not satisfactory and there is room for improvement. Computational models based on machine learning approaches may further enhance prediction performance as well as provide effective large-scale analysis of available clinical data. The final prediction performances of the 5-fold CV and external validation tests were obtained by averaging the 100 corresponding performances of the internal and external sets, respectively. Furthermore, an independent dataset (Table 6) was used to assess the true predictive power of our proposed discriminating model.

As noticed in Table 6, the four or five prediction models give the prediction results on samples 17 and 25 as TT case, but the correct result is IDA case. These results might be due to the distribution of samples 17 and 25 is close to TT case, as illustrated in Fig. 6. Another reason supported our results is that thalassemia and IDA are most common etiologies of hypochromic microcytic anemia in Thailand therefore, both of them are close differential diagnosis and can coexist together. Prior studies showed that there is frequent occurrence of IDA in TT [60, 61]. Interestingly, this finding inspires us to perform future research for construction new efficient model to distinguish three groups including TT, IDA and coexisting of these two conditions.

As shown in Table 4, SVM and RF models perform well with the highest and second highest prediction results, respectively, for correctly discriminating IDA from TT. However, SVM models do not lend themselves as easily to interpretability. SVM is classified as black box model that could work perfectly with unknown data [62] because it overcame the problem of overfitting from DT 


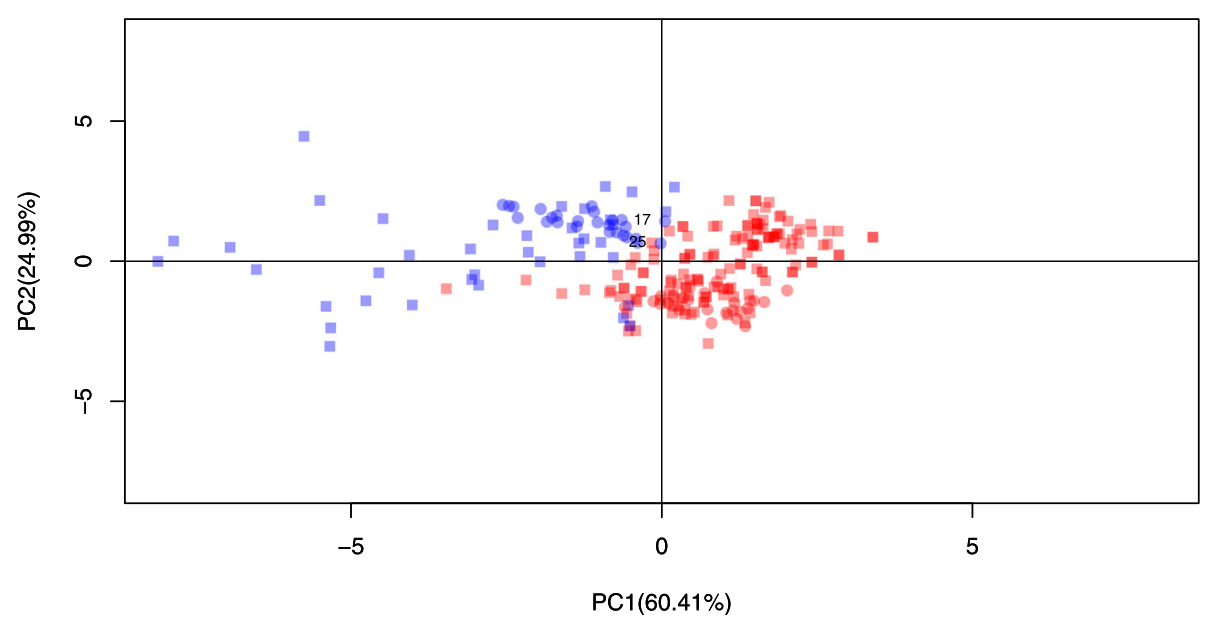

Fig. 6 Multivariate analysis using principal component analysis (PCA) of internal (square) and external (circles) sets, where red and blue represent $\Pi$ and IDA cases, respectively

[63]. According to DT model showed good performance on the training dataset but poor classification to other dataset in case the data is various detail and complex. RF could solve this limitation of DT [64]. Therefore, the seven interpretable rules (Table 5) extracting from RF were provided to represent criteria of each parameters for discrimination.

By observing the prediction results listed in Tables 3 and 4, we can clearly find that ThalPred (based on SVM model) has good discriminating power and outperforms all existing formulas and indices with the highest values of MCC (0.92), AUC (1.00) and YI (0.92). Improvements of MCC/YI with $18 \% / 10$ and $22 \% / 12 \%$ for MCC and YI, respectively, were observed when compared with the best (KF2) and second-best (RI) existing formulas. Hence, ThalPred had better generalization capability for discrimination between IDA from TT than the existing formulas and indices. Further, ThalPred developed in the web server using SVM model [65] is stable and reliable to assist health care team for discrimination. This study is based on the small size [66, 67] of IDA and performed unbalancing dataset $[68,69]$. So, the predictor may not be robust enough to apply on a very diverse dataset. As soon as more patients' data require retraining as the new independent dataset to make the predictor more robust. This suggested that partition of the patients' age for training dataset to make more sensitive and specific should be considered for the future development of effective models. The mobile application also be one choice for more convenient and available for all users.

\section{Conclusion}

Discrimination between patients with IDA and TT is still a challenging problem due to the diversity of populations with anemia. Computational models can accelerate the process of screening HMA patients and save a lot of expenses and time. In this study, we have extracted an interpretable rule and established a web-based tool for discriminating IDA from TT. The prediction results for both cross-validation and independent validation tests on our laboratory data demonstrate the superiority of ThalPred over existing indices and formulas. Furthermore, a userfriendly web-based tool for ThalPred was established at http://codes.bio/thalpred/, by which users can easily obtain the prediction result without the need to follow the mathematical and computational details. We believe that the proposed ThalPred will supplement the existing indices and formulas as well as facilitate the health care provider.

\section{Abbreviations \\ 5-fold CV: Five-fold cross validation; Ac: Accuracy; ANN: Artificial neural network; AUC: Area under receiver operating characteristics curve; CART: classification and regression tree; CE: Capillary electrophoresis; DT: Decision tree; Hb: Hemoglobin; Hct: Hematocrit; HMA: Hypochromic microcytic anemia; IDA: Iron deficiency anemia; $k$-NN: $k$-nearest neighbor; LPLC: Low pressure liquid chromatography; MCC: Matthew correlation coefficient; MCH: Mean corpuscular hemoglobin; MCHC: Mean corpuscular hemoglobin concentration; MCV: Mean corpuscular volume; PCA: Principal component analysis; PCs: Principal components; RBC: Red blood cell; RDW: Red blood cell distribution width; RF: Random forest; SD: Standard deviation; Sn: Sensitivity; Sp: Specificity; SVM: Support vector machine; TT: Thalassemia trait; Yl: Youden's Index}

\section{Acknowledgements}

We thank the editor and three anonymous reviewers for valuable comments and suggestion on our study.

\section{Authors' contributions}

All authors have read and approved of the final version of the manuscript. VL: data/machine learning analysis, result interpretation and manuscript preparation. WS: computational model, algorithm analysis, manuscript preparation and website construction. WA: correlation and interpretation of laboratory data and data processing. CN: project discussion and website construction. VP: result analysis and discussion. PN: project initiation, study 
design, correlation and interpretation of laboratory data, and manuscript preparation.

\section{Funding}

This study was supported by the Faculty of Medical Technology, Mahidol University, Office of the Higher Education Commission (OHEC) and the Thailand Research Fund to PN (MRG5460082) and to WS (MRG6180226) as well as the Ministry of Europe and Foreign Affairs (MEAE), the Ministry of Higher Education, Research and Innovation (MESRI) and the Office of the Higher Education Commission (OHEC) via the Franco - Thai Cooperation Program in Higher Education and Research (Frnco-Thai Mobility Progra/PHC SIAM) to PN. The funding body has no role in the design of the study, data collection, analysis and interpretation of data and in writing the manuscript.

\section{Availability of data and materials}

The datasets used and/or analyzed during the current study available from the corresponding author on reasonable request.

\section{Ethics approval and consent to participate}

The study was conducted under the approval of Mahidol University Central Institutional Review Board (MU-CIRB: 2016/084.0311) We received a participant consent waiver from MU-CIRB. All information of subjects was deidentified prior data analysis.

\section{Consent for publication}

Not Applicable

\section{Competing interests}

The authors declare that they have no competing interest and the research was conducted in the absence of any commercial or financial relationship that could be influenced as potential conflict of interest.

\begin{abstract}
Author details
${ }^{1}$ Center for Research and Innovation, Faculty of Medical Technology, Mahidol University, Bangkok, Thailand. ${ }^{2}$ Department of Clinical Microscopy, Faculty of Medical Technology, Mahidol University, Bangkok, Thailand. ${ }^{3}$ Center of Data Mining and Medical Informatics, Faculty of Medical Technology, Mahidol University, Bangkok, Thailand. ${ }^{4}$ Department of Clinical Microbiology and Applied Technology, Faculty of Medical Technology, Mahidol University, Bangkok, Thailand.
\end{abstract}

Received: 11 October 2017 Accepted: 14 October 2019 Published online: 07 November 2019

\section{References}

1. Kassebaum NJ, Jasrasaria R, Naghavi M, Wulf SK, Johns N, Lozano R, et al. A systematic analysis of global anemia burden from 1990 to 2010. Blood. 2014;123:615-24. https://doi.org/10.1182/blood-2013-06-508325.

2. Winichagoon P. Prevention and control of anemia: Thailand experiences. $J$ Nutr. 2002;132(4 Suppl):862S-6S.

3. Thong XY, Lee LY, Chia DA, Wong YC, Biswas A. Management and outcomes of fetal hydrops in a tertiary care centre in singapore. Ann Acad Med Singap. 2017:46:4-10

4. Johnson-Wimbley TD, Graham DY. Diagnosis and management of iron deficiency anemia in the 21st century. Therap Adv Gastroenterol. 2011;4 177-84. https://doi.org/10.1177/1756283X11398736.

5. Nathalang O, Arnutti $P$, Nillakupt K. Thalassemia screening among Royal Thai Army medical cadets. Mil Med. 2004;169:274-6. https://doi.org/10.7205/ MILMED.169.4.274

6. Nathalang O, Nillakupt K, Arnutti P, Boonsiri T, Panichkul S, Areekul W. Screening for thalassemia and hemoglobinopathy in a rural area of Thailand: a preliminary study. J Med Assoc Thai. 2005;88(Suppl 3):S35-42.

7. Ou Z, Li Q, Liu W, Sun X. Elevated hemoglobin A2 as a marker for $\beta$ thalassemia trait in pregnant women. Tohoku J Exp Med. 2011;223:223-6.

8. Bessman JD, Feinstein DI. Quantitative anisocytosis as a discriminant between iron deficiency and thalassemia minor. Blood. 1979;53:288-93.

9. Ehsani MA, Shahgholi E, Rahiminejad MS, Seighali F, Rashidi A. A new index for discrimination between iron deficiency anemia and beta-thalassemia minor: results in 284 patients. Pak J Biol Sci. 2009;12:473-5.

10. England JM, Fraser PM. Differentiation of iron deficiency from thalassaemia trait by routine blood-count. Lancet. 1973;1:449-52.
11. Green R, King R. A new red cell discriminant incorporating volume dispersion for differentiating iron deficiency anemia from thalassemia minor Blood Cells. 1989;15:481-91 discussion 492.

12. Mentzer WC. Differentiation of iron deficiency from thalassaemia trait. Lancet. 1973;1:882.

13. Jayabose S, Giamelli J, LevondogluTugal O, Sandoval C, Ozkaynak F, Visintainer $P$. Differentiating iron deficiency anemia from thalassemia minor by using an RDW-based index. J Pediatr Hematol Oncol. 1999;21(4):314.

14. Ricerca BM, Storti S, d'Onofrio G, Mancini S, Vittori M, Campisi S, et al. Differentiation of iron deficiency from thalassaemia trait: a new approach. Haematologica. 1987;72:409-13.

15. Shine I, Lal S. A strategy to detect beta-thalassaemia minor. Lancet. 1977;1:692-4.

16. Sirdah M, Tarazi I, Al Najjar E, Al HR. Evaluation of the diagnostic reliability of different RBC indices and formulas in the differentiation of the betathalassaemia minor from iron deficiency in Palestinian population. Int J Lab Hematol. 2008;30:324-30. https://doi.org/10.1111/j.1751-553X.2007.00966.x.

17. Srivastava PC, Bevington JM. Iron deficiency and-or thalassaemia trait. Lancet. 1973;1:832.

18. Sirachainan $N$, lamsirirak $P$, Charoenkwan $P$, Kadegasem $P$, Wongwerawattanakoon $\mathrm{P}$, Sasanakul W, et al. New mathematical formula for differentiating thalassemia trait and iron deficiency anemia in thalassemia prevalent area: a study in healthy school-age children. Southeast Asian J Trop Med Public Health. 2014;45:174-82.

19. Hafeez Kandhro A, Shoombuatong W, Prachayasittikul V, Nuchnoi P. New Bioinformatics-Based Discrimination Formulas for Differentiation of Thalassemia Traits From Iron Deficiency Anemia. Lab Med. 2017;48:230-7. https://doi.org/10.1093/labmed//mx029.

20. van Alphen $B, B H J$ W, Frens MA. Age- and sex-related differences in contrast sensitivity in C57BL/6 mice. Invest Ophthalmol Vis Sci. 2009;50:2451-8. https://doi.org/10.1167/iovs.08-2594.

21. Ambayya A, Su AT, Osman NH, Nik-Samsudin NR, Khalid K, Chang KM, et al. Haematological reference intervals in a multiethnic population. PLOS ONE. 2014:9:e91968. https://doi.org/10.1371/journal.pone.0091968.

22. Huang T-C, Wu Y-Y, Chen Y-G, Lai S-W, Wu S-C, Ye R-H, et al. Discrimination index of microcytic anemia in young soldiers: a single institutional analysis. PLoS ONE. 2015;10:e0114061. https://doi.org/10.1371/journal.pone.0114061.

23. Miri-Moghaddam E, Sargolzaie N. Cut off determination of discrimination indices in differential diagnosis between iron deficiency anemia and $\beta$ thalassemia minor. Int J Hematol Oncol Stem Cell Res. 2014;8:27-32.

24. Bordbar E, Taghipour M, Zucconi BE. Reliability of Different RBC Indices and Formulas in Discriminating between $\beta$-Thalassemia Minor and other Microcytic Hypochromic Cases. Mediterr J Hematol Infect Dis. 2015;7: e2015022. https://doi.org/10.4084/MJHID.2015.022.

25. Shoombuatong W, Prathipati P, Prachayasittikul V, Schaduangrat N, Malik AA, Pratiwi R, et al. Towards Predicting the Cytochrome P450 Modulation: From QSAR to proteochemometric modeling. Curr Drug Metab. 2017. https://doi.org/10.2174/1389200218666170320121932.

26. Shoombuatong W, Prachayasittikul V, Anuwongcharoen N, Songtawee N, Monnor T, Prachayasittikul S, et al. Navigating the chemical space of dipeptidyl peptidase-4 inhibitors. Drug Des Devel Ther. 2015;9:4515-49. https://doi.org/10.2147/DDDT.S86529.

27. Shoombuatong W, Prachayasittikul V, Prachayasittikul V, Nantasenamat C. Prediction of aromatase inhibitory activity using the efficient linear method (ELM). EXCLI J. 2015;14:452-64. https://doi.org/10.17179/excli2015-140.

28. Win TS, Malik AA, Prachayasittikul V, JE SW, Nantasenamat C, Shoombuatong W. HemoPred: a web server for predicting the hemolytic activity of peptides. Future Med Chem. 2017;9:275-91. https://doi.org/10. 4155/fmc-2016-0188.

29. Le S, Josse J, Husson F. FactoMineR: an R package for multivariate analysis. J Stat Software. 2008;25:1-8.

30. R Development Core Team. R: A Language and Environment for Statistical Computing. Vienna: the R Foundation for Statistical Computing; 2011. ISBN: 3-900051-07-0. http://www.R-project.org/

31. Quinlan JR. C4.5: Programs for Machine Learning. Amsterdam: Elsevier; 2014.

32. Cutler A. randomForest: Random Forests for Classification and Regression; 2006.

33. Holmes G, Donkin A, Witten IH. WEKA: a machine learning workbench. In: Proceedings of ANZIIS ' 94 - Australian New Zealand intelligent information systems conference. IEEE; 1994. p. 357-61. https://doi.org/10.1109/ANZIIS. 1994.396988. 
34. Breiman L, Friedman J, Stone CJ, Olshen RA. Classification and Regression Trees Taylor \& Francis Group: CRC Press; 1984.

35. Calle ML, Urrea V. Letter to the editor: stability of random forest importance measures. Brief Bioinform. 2011;12(1):86-9.

36. Agatonovic-Kustrin S, Beresford R. Basic concepts of artificial neural network (ANN) modeling and its application in pharmaceutical research. J Pharm Biomed Anal. 2000;22:717-27. https://doi.org/10.1016/50731-7085(99)00272-1.

37. Liu X. Deep Recurrent Neural Network for Protein Function Prediction from Sequence. BioRxiv. 2017. https://doi.org/10.1101/103994.

38. Liang M, Hu X. Recurrent convolutional neural network for object recognition. In: 2015 IEEE Conference on computer vision and pattern recognition (CVPR). IEEE; 2015. p. 3367-75. https://doi.org/10.1109/CVPR. 2015.7298958

39. Cortes C, Vapnik V. Support-vector networks. Mach.Learn. 1995;20(3):273-97.

40. Erfani SM, Rajasegarar S, Karunasekera S, Leckie C. High-dimensional and large-scale anomaly detection using a linear one-class SVM with deep learning. Pattern Recognit. 2016;58:121-34. https://doi.org/10.1016/j.patcog. 2016.03.028

41. David Meyer, Evgenia Dimitriadou, Kurt Hornik, Andreas Weingessel and Friedrich Leisch (2017). e1071: Misc Functions of the Department of Statistics, Probability Theory Group (Formerly: E1071), TU Wien. R package version 1.6-8. https://CRAN.R project.org/package=e1071

42. Motsinger AA, Ritchie MD. The effect of reduction in cross-validation intervals on the performance of multifactor dimensionality reduction. Genet Epidemiol. 2006;30:546-55. https://doi.org/10.1002/gepi.20166.

43. Baratloo A, Hosseini M, Negida A, El Ashal G. Part 1: Simple definition and calculation of accuracy, sensitivity and specificity. Emerg (Tehran). Spring. 2015;3(2):48-49

44. Parikh R, Mathai A, Parikh S, Chandra Sekhar G, Thomas R. Understanding and using sensitivity, specificity and predictive values. Indian J Ophthalmol. 2008;56(1):45-50.

45. Gorodkin J. Comparing two K-category assignments by a K-category correlation coefficient. Comput Biol Chem. 2004;28(5-6):367-74.

46. Fawcett T. An introduction to ROC analysis. Pattern Recognit Lett. 2006; 27(8):861-74.

47. Sing T, et al. ROCR: visualizing classifier performance in R. Bioinformatics. 2005;21(20):3940-1.

48. Boughorbel S, Jarray F, El-Anbari M. Optimal classifier for imbalanced data using Matthews Correlation Coefficient metric. PLoS ONE. 2017;12:e0177678. https://doi.org/10.1371/journal.pone.0177678.

49. Agrawal P, Kumar S, Singh A, Raghava GPS, Singh IK. NeuroPlpred: a tool to predict, design and scan insect neuropeptides. Sci Rep. 2019;9:5129. https:// doi.org/10.1038/s41598-019-41538-x.

50. Bramer M. Avoiding overfitting of decision trees. In: Principles of data mining. London: Springer London; 2013. p. 121-36. https://doi.org/10.1007/ 978-1-4471-4884-5_9.

51. Gupta D. Applied analytics through case studies using Sas and R: implementing predictive models and machine learning techniques. Berkeley: Apress; 2018. https://doi.org/10.1007/978-1-4842-3525-6.

52. Ntaios G, Chatzinikolaou A, Saouli Z, Girtovitis F, Tsapanidou M, Kaiafa G, et al. Discrimination indices as screening tests for beta-thalassemic trait. Ann Hematol. 2007;86:487-91. https://doi.org/10.1007/s00277-007-0302-X.

53. Okan V, Cigiloglu A, Cifci S, Yilmaz M, Pehlivan M. Red cell indices and functions differentiating patients with the beta-thalassaemia trait from those with iron deficiency anaemia. J Int Med Res. 2009;37:25-30. https://doi.org/ 10.1177/147323000903700103.

54. Piplani S, Madaan M, Mannan R, Manjari M, Singh T, Lalit M. Evaluation of various discrimination indices in differentiating iron deficiency anemia and beta thalassemia trait: A practical low cost solution. Annal Pathol Lab Med. 2016;3(6):A551-59.

55. Pornprasert S, Panya A, Punyamung M, Yanola J, Kongpan C. Red cell indices and formulas used in differentiation of $\beta$-thalassemia trait from iron deficiency in Thai school children. Hemoglobin. 2014;38:258-61. https://doi. org/10.3109/03630269.2014.930044.

56. Plengsuree S, Punyamung M, Yanola J, Nanta S, Jaiping K, Maneewong K, et al. Red Cell Indices and Formulas Used in Differentiation of $\beta$-Thalassemia Trait from Iron Deficiency in Thai Adults. Hemoglobin. 2015;39:235-9. https://doi.org/10.3109/03630269.2015.1048352.

57. Wongprachum K, Sanchaisuriya K, Sanchaisuriya P, Siridamrongvattana S, Manpeun S, Schlep FP. Proxy indicators for identifying iron deficiency among anemic vegetarians in an area prevalent for thalassemia and hemoglobinopathies. Acta Haematol. 2012;127:250-5. https://doi.org/10. 1159/000337032.

58. Lurie JD, Sox HC. Principles of medical decision making. Spine. 1999;24:493-8.

59. Krieg AF, Abendroth TW, Bongiovanni MB. When is a diagnostic test result positive? Decision tree models based on net utility and threshold. Arch Pathol Lab Med. 1986;110:787-91.

60. Verma S, Gupta R, Kudesia M, Mathur A, Krishan G, Singh S. Coexisting iron deficiency anemia and Beta thalassemia trait: effect of iron therapy on red cell parameters and hemoglobin subtypes. ISRN Hematol. 2014;2014:293216. https://doi.org/10.1155/2014/293216.

61. Mohammad N, Chowdhury MJ, Alam MR, Kabir AL, Rahaman MFU, Chakrabarty B. Co-existence of iron deficiency in beta thalassaemia trait. J Med. 2017:19:44. https://doi.org/10.3329/jom.v19i1.34842.

62. Noble WS. What is a support vector machine? Nat Biotechnol. 2006;24: 1565-7. https://doi.org/10.1038/nbt1206-1565.

63. Hawkins DM. The problem of overfitting. J Chem Inf Comput Sci. 2004;44:112. https://doi.org/10.1021/ci0342472.

64. Svetnik V, Liaw A, Tong C, Culberson JC, Sheridan RP, Feuston BP. Random forest: a classification and regression tool for compound classification and QSAR modeling. J Chem Inf Comput Sci. 2003;43:1947-58. https://doi.org/ 10.1021/ci034160g.

65. Li YH, Xu JY, Tao L, Li XF, Li S, Zeng X, et al. SVM-Prot 2016: A Web-Server for Machine Learning Prediction of Protein Functional Families from Sequence Irrespective of Similarity. PLoS ONE. 2016;11:e0155290. https://doi. org/10.1371/journal.pone.0155290.

66. Dougherty ER, Dalton LA. Scientific knowledge is possible with small-sample classification. EURASIP J Bioinform Syst Biol. 2013;2013:10. https://doi.org/10. 1186/1687-4153-2013-10.

67. Popovici V, Chen W, Gallas BG, Hatzis C, Shi W, Samuelson FW, et al. Effect of training-sample size and classification difficulty on the accuracy of genomic predictors. Breast Cancer Res. 2010;12:R5. https:// doi.org/10.1186/bcr2468.

68. Khan SH, Hayat M, Bennamoun M, Sohel FA, Togneri R. Cost-Sensitive Learning of Deep Feature Representations From Imbalanced Data. IEEE Trans Neural Netw Learn Syst. 2017. https://doi.org/10.1109/ TNNLS.2017.2732482.

69. Guo H, Zhi W, Liu H, Xu M. Imbalanced learning based on logistic discrimination. Comput Intell Neurosci. 2016;2016:5423204. https://doi.org/ $10.1155 / 2016 / 5423204$

\section{Publisher's Note}

Springer Nature remains neutral with regard to jurisdictional claims in published maps and institutional affiliations.

Ready to submit your research? Choose BMC and benefit from:

- fast, convenient online submission

- thorough peer review by experienced researchers in your field

- rapid publication on acceptance

- support for research data, including large and complex data types

- gold Open Access which fosters wider collaboration and increased citations

- maximum visibility for your research: over $100 \mathrm{M}$ website views per year

At $\mathrm{BMC}$, research is always in progress.

Learn more biomedcentral.com/submission 